\title{
EUV Emission Sources in Gas-Dynamic Models of Stellar Flares
}

\author{
M. A. LIVSHITS ${ }^{1}$ AND M. M. KATSOVA ${ }^{2}$ \\ ${ }^{1}$ Institute of Terrestrial Magnetism, Ionosphere, and Radio Wave Propagation, \\ Russian Academy of Sciences, 142092 Troitsk, Moscow Region, Russia \\ ${ }^{2}$ Sternberg State Astronomical Institute, Moscow State University, \\ 119899 Moscow, Russia
}

A stellar flare model in which the main energy release is located above the chromosphere based on a set of elementary acts of the electron acceleration or impulsive heating of plasma is discussed. The response of the chromosphere to impulsive heating for both a single burst and the simultaneous effect of a set of the bursts is considered.

The results of numerical modeling of the process of explosive evaporation of the stellar chromosphere allow us to select 3 classes of EUV emission source: (1) the bursts of the EUV emission in the temperature range of $3 \cdot 10^{4}-3 \cdot 10^{5} \mathrm{~K}$ at the beginning of each of elementary act of the energy release; (2) the bursts of the EUV emission at temperatures $T \approx 10^{6} \mathrm{~K}$, accompanied by outflow of heated plasma; (3) the EUV emission, caused by new features of the process, namely, when the maxima of the distribution of the pressure are forming in the region of the downward-moving thermal front.

The properties of the EUV sources, velocities of the plasma motions therein, and possible behaviour of the light curve for an elementary burst are discussed.

\section{Introduction}

In previous papers we concentrated on understanding the dynamics of plasma in stellar flaring processes. The acceleration of particles takes place often enough, especially in small-scale regions, and apparently there are different mechanisms that accelerate electrons up to $20-100 \mathrm{keV}$ energies. The response of the chromosphere to impulsive heating by nonthermal electrons should lead to a development of specific plasma motions: the low-temperature condensation moves downward, and the hot plasma evaporates upward. The first one emits in the Balmer lines and, in some cases, in the optical continuum; the evaporated plasma turns out to be the source of the soft X-ray emission. The modelling of gas-dynamical processes helps us to interpret the flare light curves in the optical and X-ray ranges, and to estimate the area of the source of the optical continuum and other characteristics of the emission sources (see details in Katsova \& Livshits 1995 and references therein).

Here we would like to discuss the properties of the sources of EUV emission formed during the gas-dynamical process referred to above. We will mainly discuss the process of explosive evaporation which is realized during impulsive flares on red dwarf stars. Gentle evaporation can appear to develop on late-type subgiants and giants, and it will be accompanied by the EUV emission of stellar wind and long-duration flares. However, we are at the beginning of understanding these problems.

\section{The Source of EUV Emission at the Start of the Gas-Dynamical Process}

Recently we carried out new modelling of this gas-dynamical process using modern numerical methods (Boiko \& Livshits 1995; Katsova et al. 1995). The system of equa- 
tions of the gas-dynamics for $2 T$ - and one-fluid plasmas includes heating flux, radiative energy losses, thermal conduction flux, and the function of the energy change between the electron and ion plasma components. In the first tenth of a second the upper chromosphere is heated suddenly without a remarkable change in the total density, because bulk motions are still practically absent. The temperature turns out to be constant in these layers of the upper chromosphere because the heating has been compensated for by radiative losses, and this value of $T$ varies from $\approx 10^{4}$ to $\leq 10^{5} \mathrm{~K}$. These layers (the source I in Figure 1) emit in the UV-lines with $\lambda=1000-2000 \AA$, and in the resonance line of He II with $\lambda=304 \AA$. This emission appears after each elementary act of an acceleration, and lasts less than one second. The same type of emission should arise when the thermal wave arrives at the second footpoint of the large-scale coronal loop.

The UV and EUV burst should be shorter than one second (see Fisher 1987 for the case of solar flares, and Katsova \& Livshits 1989 for the flares on red dwarfs). The burst in the He II line can be longer than $1 \mathrm{~s}$ because the ionization of $\mathrm{He} \mathrm{I}$ by the EUV and soft X-ray emission can be remarkable in some cases, and this effect is able to prolong the emission at $304 \AA$.

Unfortunately, there is no direct evidence for an identification of such very short UV and EUV spikes with the source arising in the beginning of the process of explosive evaporation. Our interpretation (Katsova \& Livshits 1989) of observations of a very short burst in the C IV line, detected on EV Lac by the ASTRON (Burnasheva et al. 1989) showed that this is possible, but it supposes that the response of the chromosphere to the heating extends over a large area. This enlargement of the flare area is understandable, if we consider the more complicated scenario of an impulsive stellar flare like that proposed by Katsova \& Livshits (1992).

\section{The EUV Emission of the Evaporated Plasma}

The computations give $5 \cdot 10^{19}$ for the number of protons that evaporate per $\mathrm{cm}^{2}$ during one elementary burst lasting $10 \mathrm{~s}$. The main part of the evaporated plasma has a temperature of $T \geq 10^{7} \mathrm{~K}$ and a velocity close to $1000 \mathrm{~km} / \mathrm{s}$. However, the temperature of evaporated plasma reaches only $(1-4) \cdot 10^{6} \mathrm{~K}$ in the beginning of this process, especially in the lower part of the upward flow. This is shown in Figure 1 for the time $t=0.4$ $\mathrm{s}$. Note that in this moment $30 \%$ of the hot plasma moves downward with velocities of $100-180 \mathrm{~km} / \mathrm{s}$, and the remaining $70 \%$ of the gas moves upward with velocities from a few $\mathrm{km} \mathrm{s}^{-1}$ up to $1000 \mathrm{~km} / \mathrm{s}$.

Thus, the plasma with temperature $(1-4) \cdot 10^{6} \mathrm{~K}$ in the lower part of the evaporated flow is the source of the EUV-lines for the few seconds after the beginning of the process. These lines have to be broaded by the Doppler effect, but they shouldn't have remarkable blueshift. The blueshifted components of high temperature lines observable during flares are those emitted by ions such as Fe XXIV and $\mathrm{Ca}$ XIX. The intensities of the EUV lines depend on the area of a flare, which is close to the area of the optical continuum source. The rise of the intensity of these lines is 1-2 $\mathrm{s}$ ahead of the appearance of the optical continuum, and is about $30 \mathrm{~s}$ ahead of the soft $\mathrm{X}$-ray radiation during powerful events with duration not longer than a few minutes; such events can be considered a response to a single act of primary energy release. 

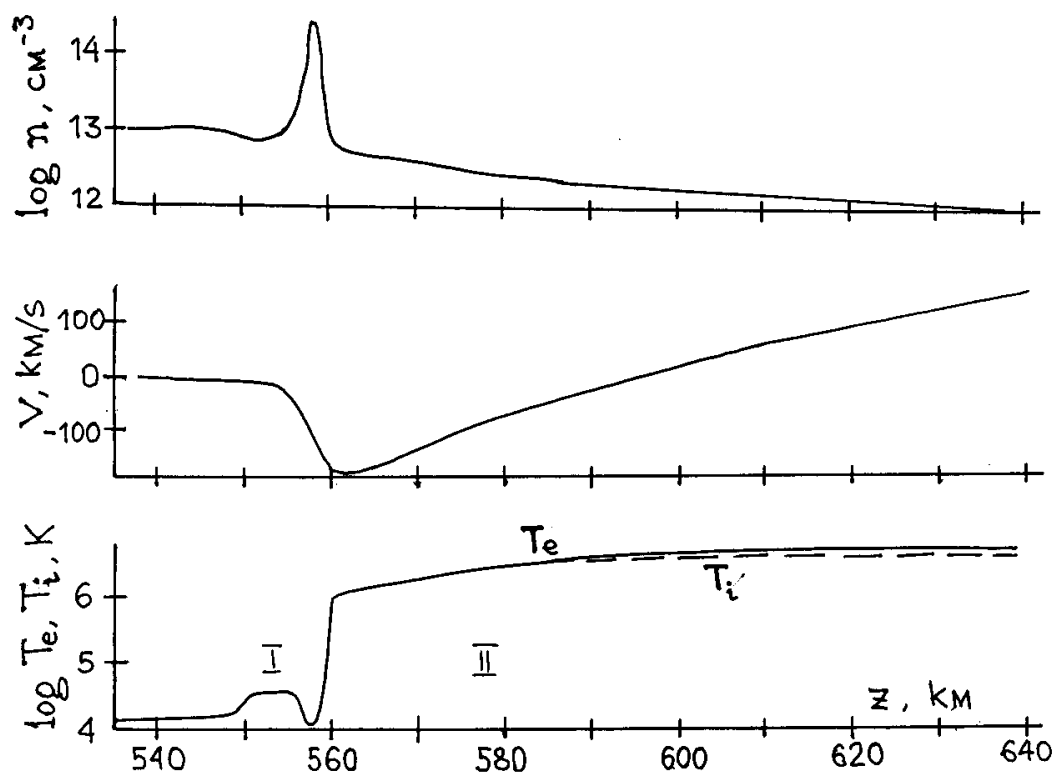

FIGURE 1. The density, velocity and temperature distributions versus height in the AD Leo atmosphere through $0.4 \mathrm{~s}$ after the beginning of heating, when the radiative shock wave has just been formed. The zero-point for the height is the photospheric level with $\tau(5000)=1$. The origin of the EUV source (I) is caused by the initial heating of the upper chromosphere. The source (II), i.e., the low layers of evaporated plasma, exists for a few seconds after the beginning of heating.

\section{The Feat ures of Explosive Evaporation and the UV and EUV Radiation}

In addition to the previously discussed possibilities for the appearance of the UV and EUV emission, there is another possibility caused by the character of the propagation of the downward thermal wave front. A quasi-stationary regime of the flow is almost always set, if powerful impulsive heating exists. It takes place $0.5-1 \mathrm{~s}$ after the beginning of the process. The character of the flow, i.e., the downward motion of the thermal wave front with a radiative shock wave ahead of it, holds until the heating operates.

In real processes going on in stellar atmospheres the temperature jump will break down, if this region is exposed to the external flow of the plasma or to another disturbance. The downward thermal front will be strongly changed when heating is varied significantly or when this thermal wave encounters some inhomogeneities of density. These events should occur with additional UV and EUV radiation.

Furthermore, the gas-dynamical modelling showed that even with no external disturbance of the quasi-steady flow, the propagation of the temperature jump downward takes place not continuously, but like a set of weak jumps (Boiko \& Livshits 1995). As a rule, the pressure is kept constant on the front of the thermal wave, i.e., the density is inversely proportional to the temperature. To simplify the gas-dynamical computations, one adopts a constant pressure for the thermal wave front. In the calculations by Boiko $\&$ Livshits (1995) no assumptions were made in this region. These results were obtained 
for continual heating. In this version of gas-dynamical simulation, the small maximum of the pressure distribution is forming in the region of the downward-moving thermal wave front from time to time. Thereafter this maximum begins to break down, and two weak disturbances start to propagate upward and downward. This brings the gas-dynamical process back to the original regime with a smooth shape for the pressure ( $p=$ const on the thermal wave front). This event is repeated time and again, and some kind of periodic process arises.

The process of the restoration of this quasi-stationary regime of the flow is accompanied by enhancement of the UV and EUV emission. The flux at $1-2000 \AA$ is close to $10^{6}$ ergs $\mathrm{cm}^{-2} \mathrm{~s}$ when explosive evaporation takes place. As a result of this effect the flare luminosities in the X-ray, UV, and optical ranges should approach each other.

The authors would like to thank the International Science Foundation for the financial support of their participation in this Colloquium. The work of M.A.L. is partly supported by the grant 4-129 of the State Program "Astronomy" of the Russian Ministry of Science.

\section{REFERENCES}

Borko, A. YA., \& Lrvshits, M. A. 1995, Astronomy Reports of the Russian Academy of Sciences, 39(3)

Burnasheva, B. A., Gershberg, R. E., Zvereva, A. M., et Al. 1989, Sov. Astron., 33, 165

FISHER, G. 1987, ApJ, 317, 502

Katsova, M. M., BoIKo, A. YA., \& Livshits, M. A. 1995, in preparation

Katsova, M. M. \& Livshits, M. A. 1989, Sov. Astron., 33, 155

Katsova, M. M. \& Livshits, M. A. 1992, Astron. Astrophis. Trans., 3, 67

Katsova, M. M. \& Livshits, M. A. 1995, in Flares and Flashes. IAU Colloq. 151, ed. J. Greiner, H. M. Duerbeck, R. E. Gershberg, Lect. Notes in Physics, Springer, 454, 177 